\title{
Analysis of the very large G-protein coupled receptor gene (Vlgr1/Mass1/USH2C) in zebrafish ${ }^{\star 2}$
}

\author{
Yann Gibert ${ }^{\mathrm{a}}$, D. Randy McMillan ${ }^{\mathrm{b}}$, Kathleen Kayes-Wandover ${ }^{\mathrm{b}}$, Axel Meyer ${ }^{\mathrm{a}}$, \\ Gerrit Begemann ${ }^{\mathrm{a}}$, Perrin C. White ${ }^{\mathrm{b}, *}$ \\ a Department of Biology, University of Konstanz, 78457 Konstanz, Germany \\ ${ }^{\mathrm{b}}$ Department of Pediatrics, University of Texas Southwestern Medical Center, 5323 Harry Hines Boulevard, Dallas, Texas 75390-9063, USA
}

Received 29 September 2004; received in revised form 6 March 2005; accepted 4 May 2005

Received by M. Schartl

\begin{abstract}
Very Large G-protein coupled Receptor-1 (VLGR1/Mass1/USH2C) is the largest known cell surface protein in vertebrates. Mutations in VLGR1 are associated with audiogenic epilepsy in mice and Usher syndrome (sensorineural deafness and retinitis pigmentosa) in humans. We characterized the zebrafish VLGR1 gene ( $v \lg r 1)$. It is $51 \%$ identical to human VLGR1 in amino acid sequence, but is $64 \%$ identical in the 7-transmembrane and cytoplasmic domains. It is 6199 amino acids in size and is encoded by a $19.2 \mathrm{~kb}$ mRNA. All introns correspond in location and phase to those of the human and mouse genes. In situ hybridization studies of zebrafish embryos demonstrate $v$ lgr 1 expression in the developing central nervous system, particularly in the hypothalamus, epiphysis and in the rhombic lips. Expression in the eye is associated with the optic nerve. Further studies using zebrafish may help ascertain the role of Vlgr1 in neural development.
\end{abstract}

Keywords: Usher syndrome; In situ hybridization; Diencephalon; Optic nerve; Zebrafish

\section{Introduction}

G protein-coupled receptors (GPCR) are the largest known family of related proteins, comprising more than 1000 known genes. By recognizing a wide range of chemical ligands and other stimuli, including proteins, peptides, small organic molecules, proteolytic activity, ions and light (Ji et al., 1998), GPCR provide cells with much of their ability to sample and respond to their environment.

All GPCR have seven transmembrane (7-TM) segments and can be classified into several families that have

\footnotetext{
Abbreviations: VLGR1, Very Large G-protein coupled Receptor-1; GPCR, G protein-coupled receptors; RACE, rapid amplication of cDNA ends; hpf, hours-post-fertilization.

is The sequence reported in this paper has been submitted to GenBank under Accession Number AY540131.

* Corresponding author. Tel.: +1 214648 3501; fax: +1 2146489772.

E-mail address: perrin.white@utsouthwestern.edu (P.C. White).
}

significant amino acid similarity within but not between families. Family 1 (or A) includes the rhodopsins, adrenergic and dopaminergic receptors and receptors for other small organic ligand; Family 2 (or B) consists mostly of receptors for peptide hormones such as secretin, and Family 3 (or C) consists of metabotropic glutamate receptors as well as the extracellular calcium sensor (Bockaert and Pin, 1999).

Members of a recently defined subdivision within Family 2 (Stacey et al., 2000) have large ectodomains composed of tandemly repeated units such as cadherin, thrombospondin, or EGF repeats. Many of these putative GPCR are expressed in the brain, and several have apparent functions in development. For example, Flamingo specifies ventraldorsal planar cell polarity in Drosophila and its vertebrate homolog Celsr1 is involved in dendritic maintenance and growth (Usui et al., 1999; Gao et al., 2000; Shima et al., 2004), whereas latrophilin may play a role in synaptogenesis (Sudhof, 2001). 
At $\sim 6300$ amino acid residues, Very Large G-protein coupled Receptor-1 (VLGR1, MASS1) is the largest member of this subfamily and indeed, is the largest known cell surface protein (McMillan et al., 2002). VLGR1 has a very large ectodomain of unique structure, consisting mostly of 35 copies of a repeat motif of $\sim 120$ amino acid residues (Nikkila et al., 2000), termed a Calx- $\beta$ motif. (Schwarz and Benzer, 1997).

VLGR1 is expressed at high levels in the developing mouse central nervous system, particularly in the ventricular zone, which is the site of neurogenesis for the developing cortex. Expression decreases in concert with the narrowing of the ventricular zone and termination of neurogenesis (McMillan et al., 2002). Mutations in VLGR1 have functional consequences in the nervous system including audiogenic seizures in mice (Skradski et al., 2001); (McMillan and White, 2004), febrile and afebrile seizures in humans (Nakayama et al., 2002) and Usher syndrome (sensorineural deafness and retinitis pigmentosa) in humans as well (Weston et al., 2004). Thus, VLGR1 may play an important role in development of the vertebrate nervous system.

Because the zebrafish is an attractive model organism for developmental studies, we characterized the gene structure and expression of zebrafish $v \lg 1$.

\section{Methods}

\subsection{Molecular cloning}

Using the predicted human VLGR1 amino acid sequence (GenBank accession), TBLASTN was used to search zebrafish genome (http://www.ensembl.org/Danio_rerio) and EST (http://www.ncbi.nlm.nih.gov/BLAST) sequence databases. An initial search in October 2001 yielded a single whole genome shotgun trace sequence corresponding to exon 18 of the human gene, and a single cDNA clone from adult zebrafish brain corresponding to part of exon 79, all of exons 80-82, and part of exon 83. These sequences were extended in the $5^{\prime}$ and $3^{\prime}$ directions using rapid amplification of cDNA ends (RACE) on mRNA from 24 hours-postfertilization (hpf) zebrafish embryos (Fig. 1, Table 1). RACE was performed using the 'Advantage RT-for-PCR' and 'SMART RACE cDNA Amplification' Kits (Clontech). The presumed 3'-end of the mRNA was reached in a single round of 3'-RACE, whereas the presumed 5'-end of the cDNA was reached in two rounds of 5'RACE.

By January 2002, zebrafish whole-genome trace sequences (www.ncbi.nlm.nih.gov) were detected containing 21 exons (Fig. 1). The complete sequence of zebrafish vlgr 1 cDNA was obtained by amplifying overlapping fragments of cDNAs corresponding to exons $11-34,29-43,43-65,52-$ 70 and 70-85 (Table 1) using Clontech Advantage 2 reagents.

\subsection{Sequence comparisons}

Whole-genome sequence databases were searched using the human VLGR1 reference sequence (NP_115495) and the BLASTP program as implemented at www.ncbi.nlm. nih.gov/sutils/genom_table.cgi or (for chicken) www.ncbi. nlm.nih.gov/genome/seq/GgaBlast.html. The chicken genome was also searched using BLAT at genome.ucsc.edu. The database for Tetraodon nigroviridis is also available at www.genoscope.cns.fr and the database for Takifugu rubripes at www.ensembl.org.

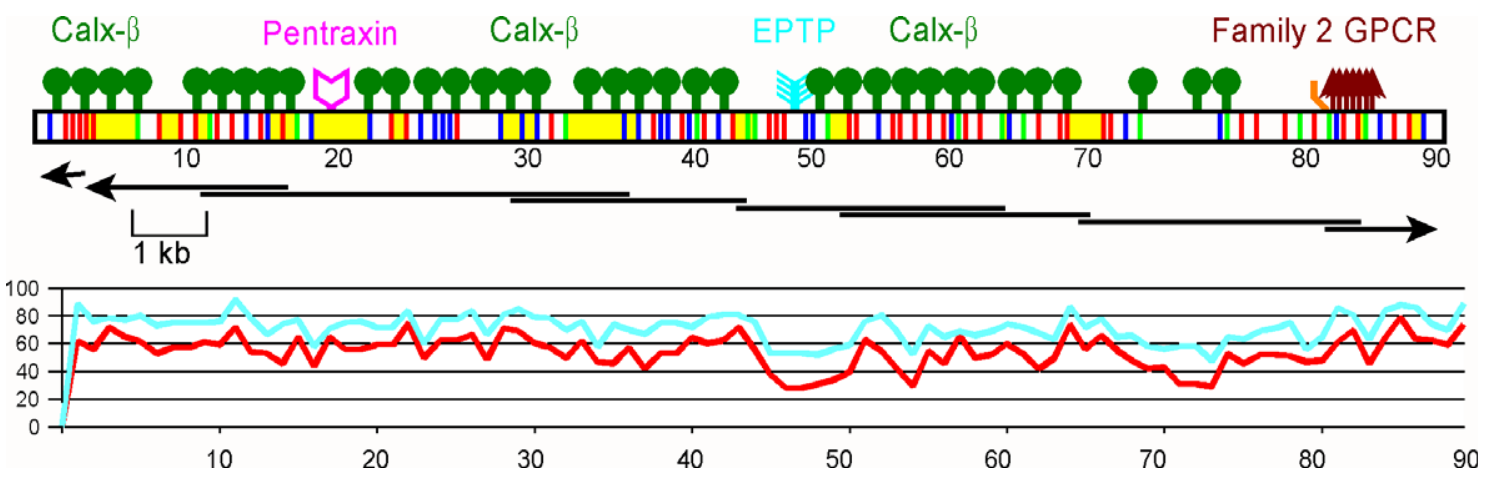

Fig. 1. Structure of $v \lg 1$ and its cDNA. The cDNA (depicted by the long rectangle) is numbered every tenth exon. The narrow vertical lines within the rectangle represent intron locations; the phase of each intron relative to the reading frame (frame 0 refers to introns located between codons) is denoted by color $(0=$ red; $1=$ blue; $2=$ green). Note that there is no obvious pattern to the location of introns with respect to the Calx- $\beta$ repeats. Yellow shading denotes exons that could be identified by running TBLASTN on the whole genome shotgun trace database using the predicted human VLGR1 amino acid sequence. Primers were selected from these sequences and used to amplify segments of $v$ lgr 1 cDNA in RT-PCR reactions using mRNA from $24 \mathrm{~h}$ zebrafish embryos (black lines below the cDNA diagram; arrows represent segments amplified by RACE). Predicted structural features of the Vlgrl protein are diagrammed at the top of the figure. The ectodomain consisting mostly of 35 Calx- $\beta$ repeats (dark green circles). There are also a single pentraxin homology domain (lavender chevron) and EPTP/ EAR motif (blue feather). The cluster of 7 brown triangles represents the region encoding the seven putative membrane-spanning segments. This receptor is in the secretin family (family 2 or B) of GPCRs, and falls within a subfamily in which all members have large (but unrelated) ectodomains. All members of this family share a G-protein coupled receptor Proteolytic Signal (GPS, denoted by the bent orange line) at which the ectodomain can be cleaved. The graph depicts percent amino acid identity (red) and similarity (blue) between human and zebrafish VLGR1, compared by individual exons. Note that exon 1 in vlgr 1 is untranslated; the initiation codon in vlgrl corresponds to Met-9 in human VLGR1. 
Table 1

Oligonucleotides used in amplifying zebrafish $v \lg r 1$ cDNA

\begin{tabular}{|c|c|c|c|}
\hline Location $^{\text {a }}$ & Exon & & Purpose \\
\hline $503-478$ as & 3 & AGTGCAGTGACATTAATGGGATCTCC & $5^{\prime} \mathrm{RACE}$ \\
\hline $445-424$ as & 3 & TGCTGCTCTCGTTCACAACAAA & 5'RACE nested \\
\hline $2394-2419 s$ & 11 & CAAAGAGGATGTCACTAAGGGAGATA & Exons $11-20,11-34$ \\
\hline $3673-3648$ & 18 & GAGCTGAATCCAGAGAGAAAATGCCA & $5^{\prime} \mathrm{RACE}$ \\
\hline $3624-3601$ & 18 & CACCACTGTAGCAGTAATTTGACC & 5'nested RACE \\
\hline $3955-3978 s$ & 20 & AAACTGGCTAACAAGCACTCAAT & Exons $20-28$ \\
\hline $4380-4357$ as & 20 & CTTCAGCCAGACAGACAGAGTGAA & Exons $11-20$ \\
\hline $6479-6456$ as & 28 & TCATTATCATCAAGCACCCTCAGG & Exons $20-28$ \\
\hline $6704-6727 s$ & 29 & TTGGAGGGATTTTTGCTGATGTGT & (83 bp deletion) \\
\hline $6823-6847 \mathrm{~s}$ & 29 & AAGCCAGTCCCCATCCTCATTATCA & Exons $29-43$ \\
\hline $6848-6868 s$ & 30 & TCAATGATGTGGTGCCAGAG & Exons $30-37$ \\
\hline $7577-7557$ as & 33 & TTTGTGCTTGTGGTGATGTTGATG & (83 bp deletion) \\
\hline $8388-8365$ as & 34 & CACCACCCCTGCTGCGTTGTCATT & Exons $11-34$ \\
\hline $8770-8751$ as & 37 & ATGACGGGGCGATGCTGAG & Exons $30-37$ \\
\hline $9708-9684$ as & 43 & AАCTCCATCCTCAAGCACCACAAAG & Exons $29-43$ \\
\hline $9674-9700 s$ & 43 & СTTCTTCTGGCTTTGTGGTGCTTGAG & Exons $43-65$ \\
\hline $11085-11109 s$ & 52 & TGTAATTGACGACGATGTTCCTGAA & Exons $52-70$ \\
\hline $13467-13443$ as & 65 & GATACCCTCAGCATTGTCATTTTTC & Exons $43-65$ \\
\hline $14610-14634 s$ & 70 & ACAACTCATTATCAGCGTCAACAGG & Exons $70-85$ \\
\hline $14787-14760$ as & 70 & CACCTGGCTACTAACTGGGACTCTAAT & Exons $52-70$ \\
\hline $17282-17307 \mathrm{~s}$ & 80 & CGTTCATAGGCAAAAACACAGACACG & $3^{\prime} \mathrm{RACE}$ \\
\hline $17543-17566 s$ & 81 & GAGGAAAACCAAAACCGAGCCAAT & 3'RACE nest \\
\hline $18106-18082$ as & 85 & CCAGCAGGACCACAACCAGAACAAT & Exons $70-85$ \\
\hline
\end{tabular}

a Starting and ending nucleotides for each primer are with reference to full-length cDNA sequence (AY540131), with 's' and 'as' indicating sense and antisense primers, respectively.

Sequences were aligned using ClustalW (Chenna et al., 2003) available commercially within the ALIGNX program in the Vector NTI suite (Informax) or via several web servers including srs.bioasp.nl:4081/cgi-bin/clustal.cgi. Phylogenetic trees were constructed using the neighborjoining algorithm (Saitou and Nei, 1987) as implemented within ALIGNX or the SRS server previously cited; other programs for phylogenetic analysis including PROTDIST, KITSCH and DRAWTREE within the PHYLIP package of programs (Kuhner and Felsenstein, 1994) were accessed on the Pasteur Institute server at bioweb.pasteur.fr/seqanal/ phylogeny/phylip-uk.html. Human (NP_115495), mouse (AAL30812), zebrafish (this work, available as NP_001001845), chicken (XP_429120.1) and Tetraodon (CAG00389.1) amino acid sequences were aligned without further modification. The predicted sequence for Takifugu rubripes was constructed by joining and/or merging the following records: SINFRUP00000084117, SINFRUP00000084116, SINFRUP00000164895, SINFRUP00000061373, SINFRUP00000061372, SINFRUP00000063310.

\subsection{Fish stocks}

Breeding zebrafish of the London wild type and the Konstanz wild type strains were reared at $28.5{ }^{\circ} \mathrm{C}$ and staged as described (Kimmel et al., 1995). The development of endogenous pigments was inhibited by exposing embryos to 1-phenyl-2-thiourea (PTU) at a final concentration of 0.2 $\mathrm{mM}$. The embryo in Fig. 3C was obtained from Scientific
Hatcheries (Huntington Beach, CA) and was not PTUtreated.

\subsection{In situ hybridization and photography}

We performed whole-mount in situ hybridization of zebrafish embryos according to standard procedures (Hauptmann, 1999) using cRNA probes labeled with digoxigeninUTP. In all but Fig. 3C, the probe consisted of nt $6848-$ 8770 (exons 30-37) of $v \lg 1$ mRNA amplified by RT-PCR on first strand cDNA prepared from $24 \mathrm{hpf}$ wild type embryos using a kit (Gibco BRL) and oligonucleotide primers: 6848-6868s; 8770-8751as (Table 1). PCR conditions were: 35 cycles of $30 \mathrm{~s}, 94{ }^{\circ} \mathrm{C} ; 30 \mathrm{~s}, 55^{\circ} \mathrm{C} ; 2 \mathrm{~min}, 72$ ${ }^{\circ} \mathrm{C}$ followed by an extension step of $7 \mathrm{~min}$ at $72{ }^{\circ} \mathrm{C}$. The resulting amplified fragment was cloned into pCR II (Invitrogen) and sequenced with universal primers for insert sequence checking. The antisense probe was generated using the SP6 promoter. Hybridization was detected with alkaline phosphate-conjugated anti-digoxigenin antibody followed by incubation with nitroblue tetrazolium and BCIP (5-bromo-4-chloro-3-indolyl phosphate).

For the experiment displayed in Fig. 3C, hybridized and stained 26 hpf embryos were formalin-fixed, embedded in paraffin and sectioned. To maximize the hybridization signal, the probes included $\sim 8 \mathrm{~kb}$ of $v \operatorname{lgr} 1$ cRNA transcribed from four different clones containing exons 11-20 (primers 2394-2419s; 4380-4357as), 20-28 (3955$3978 \mathrm{~s} ; 6479-6456 \mathrm{as}), 29-43 \quad(6823-6847 \mathrm{~s} ; 9708-$ 9684as), and 52-70 (11085-11109s; 14787-14760as), 
respectively (Table 1). Labeling was performed using reagents from Roche Applied Science (Indianapolis, IN).

Stained embryos were examined with a Zeiss Axiophot microscope. Images were processed using Zeiss Axiovision and Adobe Photoshop software.

\section{Results and discussion}

\section{1. cDNA structure and predicted protein sequence}

Zebrafish $v \lg r 1$ is encoded by a $19.2 \mathrm{~kb}$ mRNA with 5 and 3-untranslated regions of 324 and $259 \mathrm{nt}$, respectively. It is 6199 amino acids in size, differing from human VLGR1 mainly in utilizing an initiation codon in exon 2 corresponding to Met-9 of the mammalian protein, and in being 89 amino acids shorter in a portion of the ectodomain (not including a Calx- $\beta$ repeat) encoded within exon 74 . It is $51 \%$ identical to human VLGR1 in amino acid sequence, but is $64 \%$ identical in both the seven-transmembrane and cytoplasmic domains (Fig. 1).

The high degree of conservation of the putative Cterminal cytoplasmic suggests that it contains regions of functional importance, but these remain to be identified. The C-terminal residues of Vlgr1 correspond to the consensus motif (Ser/Thr)-Xaa-(Val/Ile/Leu) recognized by proteins bearing PDZ domains (Songyang et al., 1997). Such proteins act as scaffolds for assembling signal transduction proteins into functional signaling units (Tsunoda et al., 1997). We found that the C-terminus of human VLGR1 indeed interacts with two PDZ domain proteins, whirlin and harmonin, in yeast two-hybrid experiments (unpublished observations).

Most structural features previously identified in the mammalian VLGR1 ectodomain (Nikkila et al., 2000; McMillan et al., 2002) are conserved in Vlgr1. These include all 35 Calx- $\beta$ repeats or portions thereof. A region similar to a pentraxin (PTX) domain is located between residues 1324 and 1500. Such domains have a threedimensional structure closely resembling those of legume lectins (Emsley et al., 1994) and are presumed to function as interaction domains. In mammalian VLGR1, residues 3198-3532 (human numbering) form a series of seven repeats of a different motif of $\sim 44$ residues; the resulting domain is predicted to form a seven-bladed "beta propeller." Because a similar domain is found in the LGI1 gene, which is mutated in autosomal dominant partial epilepsy with auditory features (ADPEAF), it has been suggested that this domain serves a common function in these two otherwise dissimilar proteins (Scheel et al., 2002; Staub et al., 2002). It is therefore termed "the epilepsy associated repeat" (EAR) (Scheel et al., 2002) or "epitempin" (EPTP) (Staub et al., 2002) domain. However, it is rather poorly conserved in zebrafish Vlgr1 (30\% identity to human), suggesting that it may not have the same function as in the mammalian protein.

\subsection{Gene structure}

The current whole genome sequence database permits localization of all of the expected 89 introns (missing splice donors 21 and 29 and splice acceptor 20). All introns
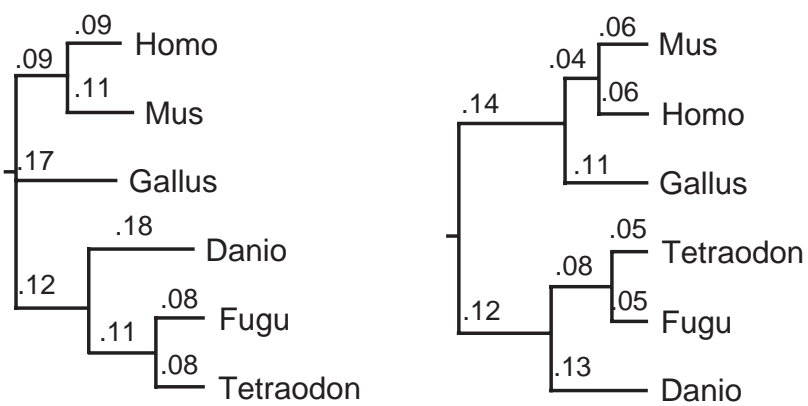

\begin{tabular}{|l|l|l|l|l|l|l|}
\hline & $\begin{array}{l}\text { Danio } \\
\text { rerio }\end{array}$ & $\begin{array}{l}\text { Homo } \\
\text { sapiens }\end{array}$ & $\begin{array}{l}\text { Mus } \\
\text { musculus }\end{array}$ & $\begin{array}{l}\text { Tetraodon } \\
\text { nigroviridis }\end{array}$ & Fugu rubripes & Gallus \\
Danio rerio & & 52 & 51 & 61 & 63 & 53 \\
Homo sapiens & 18 & & 80 & 48 & 49 & 64 \\
Mus musculus & 18 & 9 & & 47 & 49 \\
Tetraodon & 14 & 17 & 17 & & 81 & \\
nigroviridis & & 18 & 17 & 6 & & \\
Fugu rubripes & 15 & 18 & 14 & 16 & 16 & \\
Gallus gallus & 16 & 13 & & & \\
\hline
\end{tabular}

Fig. 2. Comparison of VLGR1 predicted protein sequences. Top, phylogenetic trees. Numbers represent expected branch lengths (i.e., probability of amino acid substitution at each position relative to the common ancestor). Left, entire proteins were aligned and a phylogenetic tree generated using the neighbor joining algorithm (Saitou and Nei, 1987). Right, the 7-transmembrane domains were aligned and a phylogenetic tree generated using the Fitch-Margoliash leastsquares method (Kuhner and Felsenstein, 1994). The tree on the left is unrooted; using midpoint rooting gives the same appearance as the tree on the right. Bottom, matrix of amino acid similarity (above the diagonal) and divergence (below the diagonal) for the entire VLGR1 sequence in the six organisms examined. 
correspond in location and phase to those of the human and mouse genes.

Fish genes are usually more compact than their mammalian homologs (McLysaght et al., 2000; Vinogradov, 1999). Thus far, 65/89 complete introns are in the zebrafish genome database. These are significantly smaller than the corresponding human introns (mean $\pm \mathrm{SE}, 2.0 \pm 0.5$ vs. $6.5 \pm 1.7 \mathrm{~kb}$ ), which may have functional implications. Human VLGR1 has several splice and transcriptional variants (McMillan et al., 2002) that cannot arise in Vlgr1. The most frequent of these involves exon 31, which is often aberrantly spliced in humans or mice so as to eliminate 83 nucleotides. The cryptic splice donor is not present in vlgr1, and indeed RT-PCR detects no variation in the size of this intron (primers in Table 1, data not shown). In humans but not mice (or zebrafish), transcripts often begin in intron 64 to yield exon 65 as the first exon.

Different PCR amplifications of both genomic and cDNA as well as screening of the zebrafish whole-genome sequences identified only one copy of the vlgr1 gene. Similarly, whole-genome BLAST searches of the Tetraodon nigroviridis and Takifugu rubripes genomes revealed in each case a single nearly complete predicted transcript with $61 \%-62 \%$ predicted amino acid sequence identity with zebrafish $v \lg r 1$.

As determined by whole-genome BLAST searches, the invertebrate genomes of Drosophila melanogaster and Caenorabditis elegans do not appear to contain VLGR1homologs. This absence suggests that VLGR1 arose after the common ancestor of arthropods and nematodes diverged from the ancestor of chordates during the Vendian (preCambrian) epoch 550 million years ago (Conway, 2000) or, like other genes previously thought to be human- or vertebrate-specific, it may have been lost in these ecdysozoan model organisms (Kortschak et al., 2003). On the other hand, the complete conservation of gene organization between the fish, chicken and mammalian genes implies that this organization was established before the divergence
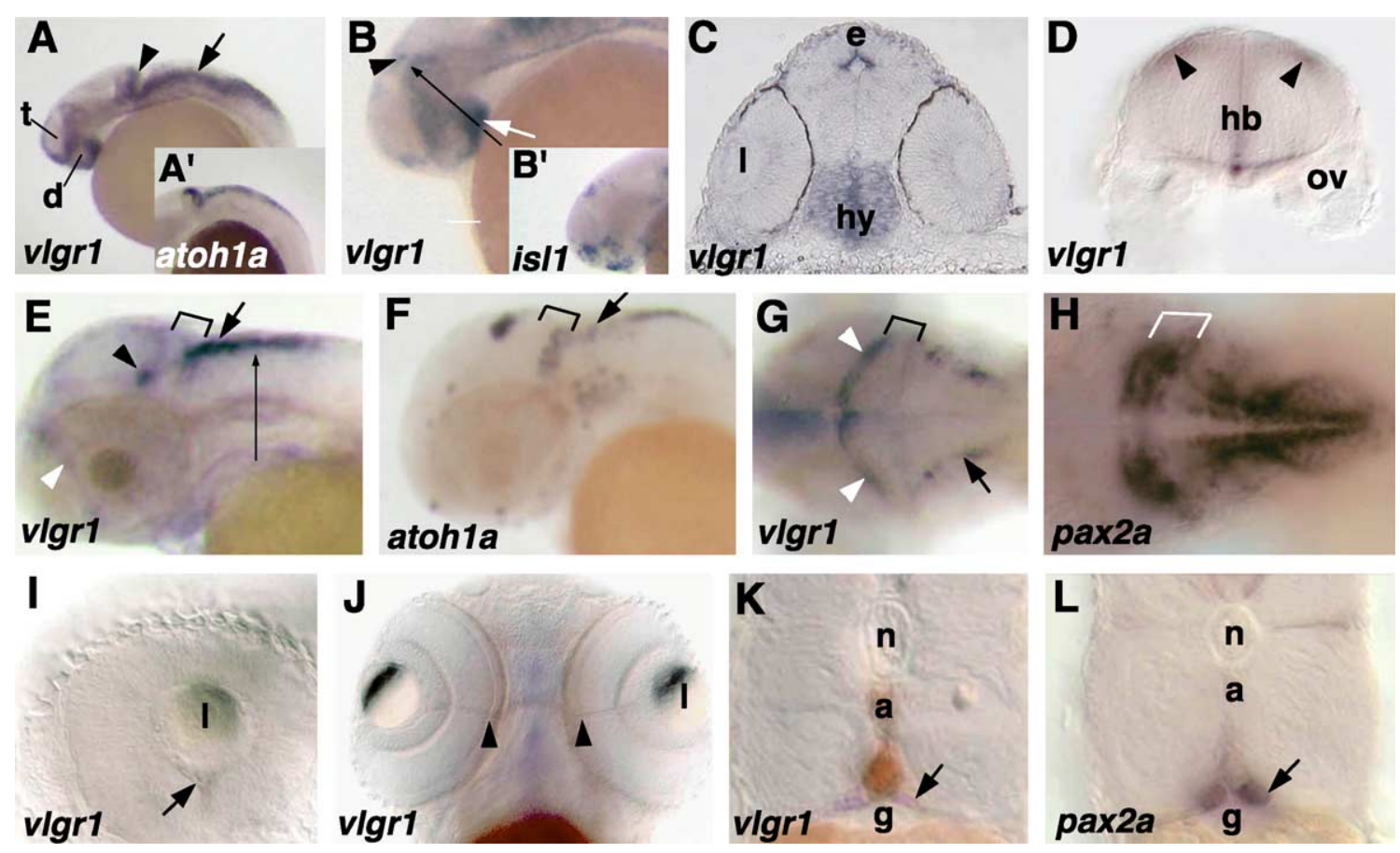

Fig. 3. Expression of $v \lg r 1$ during wild type zebrafish development. (A-E,G,I,J) Expression of $v \lg r 1$ in the central nervous system. (A) vlgrl is strongly expressed at 24 hours post fertilization (hpf) in the forebrain, at the telencephalic-diencephalic boundary and in the diencephalon; vlgr 1 expression is detected in the midbrain, and expression in the hindbrain colocalizes with that of atonal homolog la (atohla)(A'), thus identifying these domains as the upper (arrowhead) and lower rhombic lips (arrow) (Koster and Fraser, 2001). (B,C) At $26 \mathrm{hpf} v \operatorname{lgrl}$ is expressed in the hypothalamus (white arrow) and in the ventral epiphysis; compare with islet1 (isl-1) expression in the epiphysis (Korzh et al., 1993) in (B'). Elongated arrow in (B) indicates the level of the transverse section showed in (C). Dark coloration surrounding the retina (C) stems from the retinal pigmented epithelium. (D) At 48 hpf, expression is detected in the lower rhombic lip (arrowheads) in a transverse section at the level of the otic vesicle. (E) Expression at the telencephalic-diencephalic boundary (white arrowhead), in the lower rhombic lip (arrow) and in a small patch of cells (black arrowhead) in the dorsal tegmentum; comparison with atohla expression confirms their location rostral to the cerebellum (brackets in E-H) and demonstrates colocalization of vlgrl and atohla in the lower rhombic lip (arrow). Elongated arrow in (E) indicates transverse section at the level of the otic vesicles in (D). (G) Expression at $72 \mathrm{hpf}$ in the lower rhombic lip (arrow) and in the cerebellar commissure (white arrowheads). (H) pax $2 a$ expression at the same developmental stage is located to the mid-hindbrain boundary (bracket)(Thisse et al., 2001). (I,J) vlgr 1 transcript in the developing retina at $48 \mathrm{hpf}(\mathrm{I})$ and $72 \mathrm{hpf}(\mathrm{J})$; expression is first detected as weak expression in the choroid fissure (arrow in I), and at $72 \mathrm{hpf}(\mathrm{J})$ is associated with the optic nerve (arrowheads). (K,L) Transverse sections at the level of somite 10 at 72 hpf, showing $v l g r 1$ expression in the pronephric duct (arrows); compare with pronephric pax2a expression in (L); the red coloration observed is due to red blood cells in the dorsal aorta (a) and axial vein. (C,D,K,L) transverse sections; (A,B,E,F,I) lateral views; $(\mathrm{G}, \mathrm{H})$ dorsal view; (J) ventral view. Abbreviations: d, diencephalon; e, epiphysis; g, gut; hb, hindbrain; hy, hypothalamus; 1 , lens; n, notochord; ov, otic vesicle; t, telencephalon. 
of the ancestors of tetrapods and teleosts. Phylogenetic tree analysis was consistent with well-established phylogenetic divisions of tetrapods and teleosts (Fig. 2). The same topology was obtained whether the entire protein sequences were aligned or the analysis was confined to the 7transmembrane domain (amino acids 5892-6160 in the human protein). For the latter analysis, results were similar using either the Fitch-Margoliash least-squares method (Kuhner and Felsenstein, 1994) or the neighbor-joining method (Saitou and Nei, 1987); the neighbor-joining method generates an unrooted tree but the same root was obtained by midpoint rooting or using an arbitrary outgroup consisting of a distantly related Family 2 receptor (latrophilin NP_001008701, not shown).

\subsection{In situ hybridization of vlgrl cRNA to zebrafish embryos}

A 1.9-kb zebrafish vlgrl cRNA fragment containing exons 30 to 37 was amplified from $24 \mathrm{hpf}$ wild type zebrafish embryo RNA and used as an antisense probe. For embryos that were subsequently sectioned (Fig. 3C), probes totaling $\sim 8 \mathrm{~kb}$ were used. Weak $v \lg r 1$ expression was first detected at $20 \mathrm{hpf}$ in the ventral diencephalon (not shown). At 24-26 hpf, expression was prominent in the prospective fore-, mid- and hindbrain including the ventral part of the epiphysis (Fig. 3A,C). Vlgr1 expression could not be detected outside of the central nervous system (CNS) at this stage (not shown). At $48 \mathrm{hpf}$ and $72 \mathrm{hpf}$, expression was detected in the posterior dorsal tegmentum, the mid-hindbrain boundary and in the lower rhombic lip (Fig. 3D,E,G). Vlgr1 staining is associated with the optic nerve, first detected at $48 \mathrm{hpf}$ in the choroid fissure (Fig. 3I) and by 72 hpf along the optic nerve (Fig. 3J). The sensitivity of the detection method does not allow us to distinguish between expression within the optic nerve or in surrounding cells. Outside the CNS, vlgrl is expressed in the anterior pronephric duct (Fig. 3K).

\subsection{Comparison of zebrafish vlgr1 and mouse Vlgr1 expression}

The distribution of VLGR1 transcripts in the mouse has been previously analyzed (McMillan et al., 2002). Here, the analysis of a VLRG1 ortholog from zebrafish allows us to compare VLGR1 expression within the vertebrate phylum. In both species, gene expression is not detected prior to the onset of neurulation. Highest levels of Vlgr1 transcripts in mouse embryos are concentrated in the ventricular zones, where neural progenitor cells reside during neurogenesis (McMillan et al., 2002). This pattern is homologous to $v \lg r 1$ expression in the zebrafish epithelium of the third, tectal and fourth ventricles. Ventricular zones are regions of neuronal proliferation (Mueller and Wullimann, 2003), therefore the similarity between patterns of expression in mammals and fish suggests that VLGR1 might play a conserved role in neuronal proliferation. We also find apparent differences in patterns of VLGR1 expression within the nervous systems of mice and zebrafish. In zebrafish, vlgrl is strongly expressed in the ventral diencephalon (i.e., the hypothalamus). In contrast, hypothalamic expression is not particularly strong in the mouse, although persistent expression of Vlgr1 in scattered hypothalamic neurons is noted even in adult mice (McMillan et al., 2002). Conversely, Vlgr1 is expressed in the spinal cord in mice but not in zebrafish embryos.

In the mouse, Vlgr1 is expressed in both the inner layer and the outer layer of the retina at embryonic day 10.5 and in all layers of the developing eye including the lens but not in the cornea at embryonic day 15 (McMillan et al., 2002). However, staining is not associated with the optic nerve at any time in the mouse, whereas it is the only detected signal in the developing eye in the zebrafish up to $72 \mathrm{hpf}$. Thus, VLGR1 may play an additional role in development of the mammalian eye based on both its pattern of expression and on the fact that mutations in this gene cause retinitis pigmentosa associated with Usher syndrome (Weston et al., 2004).

\section{Acknowledgements}

We thank Stephen Ekker and Aubrey Nielsen (University of Minnesota) for helpful advice.

\section{References}

Bockaert, J., Pin, J.P., 1999. Molecular tinkering of $\mathrm{G}$ protein-coupled receptors: an evolutionary success. EMBO J. 18, 1723-1729 ([Review] [56 refs]).

Chenna, R., et al., 2003. Multiple sequence alignment with the Clustal series of programs. Nucleic Acids Res. 31, 3497-3500.

Conway, M.S., 2000. The Cambrian "explosion": slow-fuse or megatonnage? Proc. Natl. Acad. Sci. U. S. A. 97, 4426-4429.

Emsley, J., et al., 1994. Structure of pentameric human serum amyloid P component. Nature 367, 338-345.

Gao, F.B., Kohwi, M., Brenman, J.E., Jan, L.Y., Jan, Y.N., 2000. Control of dendritic field formation in Drosophila: the roles of flamingo and competition between homologous neurons. Neuron 28, 91-101 ([see comments])

Hauptmann, G., 1999. Two-color detection of mRNA transcript localizations in fish and fly embryos using alkaline phosphatase and beta-galactosidase conjugated antibodies. Dev. Genes Evol. 209, $317-321$

Ji, T.H., Grossmann, M., Ji, I., 1998. G protein-coupled receptors. I. Diversity of receptor-ligand interactions. J. Biol. Chem. 273, 17299-17302 ([Review] [50 refs]).

Kimmel, C.B., Ballard, W.W., Kimmel, S.R., Ullmann, B., Schilling, T.F., 1995. Stages of embryonic development of the zebrafish. Dev. Dyn. 203, 253-310.

Kortschak, R.D., Samuel, G., Saint, R., Miller, D.J., 2003. EST analysis of the cnidarian Acropora millepora reveals extensive gene loss and rapid sequence divergence in the model invertebrates. Curr. Biol. 13, $2190-2195$. 
Korzh, V., Edlund, T., Thor, S., 1993. Zebrafish primary neurons initiate expression of the LIM homeodomain protein Isl-1 at the end of gastrulation. Development 118, 417-425.

Koster, R.W., Fraser, S.E., 2001. Direct imaging of in vivo neuronal migration in the developing cerebellum. Curr. Biol. 11, 1858-1863.

Kuhner, M.K., Felsenstein, J., 1994. A simulation comparison of phylogeny algorithms under equal and unequal evolutionary rates. Mol. Biol. Evol. $11,459-468$

McLysaght, A., Enright, A.J., Skrabanek, L., Wolfe, K.H., 2000. Estimation of synteny conservation and genome compaction between pufferfish (Fugu) and human. Yeast 17, 22-36.

McMillan, D.R., White, P.C., 2004. Loss of the transmembrane and cytoplasmic domains of the very large G-protein-coupled receptor-1 (VLGR1 or Mass1) causes audiogenic seizures in mice. Mol. Cell. Neurosci. 26, 322-329.

McMillan, D.R., Kayes-Wandover, K.M., Richardson, J.A., White, P.C., 2002. Very Large G Protein-coupled Receptor-1, the largest known cell surface protein, is highly expressed in the developing central nervous system. J. Biol. Chem. 277, 785-792.

Mueller, T., Wullimann, M.F., 2003. Anatomy of neurogenesis in the early zebrafish brain. Brain Res. Dev. Brain Res. 140, 137-155.

Nakayama, J., et al., 2002. A nonsense mutation of the MASS1 gene in a family with febrile and afebrile seizures. Ann. Neurol. 52, 654-657.

Nikkila, H., McMillan, D.R., Nunez, B.S., Pascoe, L., Curnow, K.M., White, P.C., 2000. Sequence similarities between a novel putative G protein-coupled receptor and $\mathrm{Na}^{+} / \mathrm{Ca}^{2+}$ exchangers define a cation binding domain. Mol. Endocrinol. 14, 1351-1364.

Saitou, N., Nei, M., 1987. The neighbor-joining method: a new method for reconstructing phylogenetic trees. Mol. Biol. Evol. 4, 406-425.

Scheel, H., Tomiuk, S., Hofmann, K., 2002. A common protein interaction domain links two recently identified epilepsy genes. Hum. Mol. Genet. $11,1757-1762$.
Schwarz, E.M., Benzer, S., 1997. Calx, a $\mathrm{Na}-\mathrm{Ca}$ exchanger gene of Drosophila melanogaster. Proc. Natl. Acad. Sci. U. S. A. 94, 10249-10254

Shima, Y., Kengaku, M., Hirano, T., Takeichi, M., Uemura, T., 2004. Regulation of dendritic maintenance and growth by a mammalian 7 pass transmembrane cadherin. Dev. Cell Biol. 7, 205-216.

Skradski, S.L., Clark, A.M., Jiang, H.M., White, H.S., Fu, Y.H., Ptacek, L.J., 2001. A novel gene causing a Mendelian audiogenic mouse epilepsy. Neuron 31, 537-544.

Songyang, Z., et al., 1997. Recognition of unique carboxyl-terminal motifs by distinct PDZ domains. Science 275, 74-77.

Stacey, M., Lin, H.H., Gordon, S., McKnight, A.J., 2000. LNB-TM7, a group of seven-transmembrane proteins related to family-B G-proteincoupled receptors. Trends Biochem. Sci. 25, 284-289 ([Review] [46 refs]).

Staub, E., et al., 2002. The novel EPTP repeat defines a superfamily of proteins implicated in epileptic disorders. Trends Biochem. Sci. 27, 441.

Sudhof, T.C., 2001. alpha-Latrotoxin and its receptors: neurexins and Cirl/latrophilins. Annu. Rev. Neurosci. 24, 933-962 ([Review]).

Thisse, B., et al., 2001. Expression of the zebrafish genome during embryogenesis (NIH R01 RR15402). ZFIN Direct Data Submission.

Tsunoda, S., et al., 1997. A multivalent PDZ-domain protein assembles signalling complexes in a G-protein-coupled cascade. Nature 388, $243-249$.

Usui, T., et al., 1999. Flamingo, a seven-pass transmembrane cadherin, regulates planar cell polarity under the control of Frizzled. Cell 98, $585-595$.

Vinogradov, A.E., 1999. Intron-genome size relationship on a large evolutionary scale. J. Mol. Evol. 49, 376-384.

Weston, M.D., Luijendijks, M.W.J., Humphreys, K.D., Mollers, C., Kimberling, W.J., 2004. Mutations in the VLGR1 gene implicate Gprotein signaling in the pathogenesis of Usher syndrome type II. Am. J. Hum. Genet. 74, 357-366. 